\title{
ELECTROCHEMICAL IMPEDANCE SPECTROSCOPY ON THERMAL AGEING EVALUATION OF EPOXY COATING CONTAINING ZINC RICH PRIMER
}

\author{
Zalilah Sharer ${ }^{1}$, John Sykes ${ }^{2}$ \\ ${ }^{1}$ UTM-MPRC, Institute for Oil and Gas, Universiti Teknologi Malaysia, 81310, Skudai, Johor, Malaysia \\ ${ }^{2}$ Department of Materials, University of Oxford, Parks Road, OX1 3PH, Oxford, United Kingdom \\ zalilah@petroleum.utm.my,john.sykes@materials.ox.ac.uk
}

\begin{abstract}
This research concentrates on the thermal ageing of a full 3-coat system with sacrificial pigment (zinc rich) primer on mild steel where the temperature dependence test is conducted to explore the correlation between the coating resistances with the corrosion rate underneath the coating. Electrochemical impedance spectroscopy (EIS) is introduced over a range of temperature to extract activation energies for the rate of controlling processes in the corrosion reactions. It is expected that the zinc rich primer does not form a barrier coating for the metal substrate rather it will be the most active component of the substrate in the electrochemistry. Full 3-coat system with zinc rich primer show the extracted activation energy from coating resistance is significantly lower than the activation energy extracted from the charge transfer resistance. This suggested that the coating resistance from EIS cannot be controlling the corrosion reaction. The activation energies generated for the corrosion process here (78-97 $\left.\mathrm{kJmol}^{-1}\right)$ are very much higher than those of ion transport through the coating (19-37 $\left.\mathrm{kJmol}^{-1}\right)$ during early immersion. Further interesting findings come from the activation energy trends over time particularly for the corrosion process which shown that the value is decreasing where at the end of exposure, the activation energy values for coating and charge transfer resistance become quite similar. It is suggested that at this stage ion transport in the coating might be controlling the corrosion process unlike at the beginning; the activation energy is getting smaller due to coating degradation.
\end{abstract}

Index Terms: epoxy coating, electrochemical impedance spectroscopy, thermal effect, zinc rich primer

\section{INTRODUCTION}

Temperature changes impose a direct impact on the corrosive protective performance of coatings; especially in an environment where the value of temperature keeps changing. For example, a natural gas pipeline network often has continuous changes in temperature, with possible range as high as $135^{\circ} \mathrm{C}$ in the vicinity of a natural gas well to an ambient temperature at a pumping station.

Previous work $[1,2]$ has established that it could be possible to separate resistances for the coating and the coating-metal interface from EIS spectra. The plotted Arrhenius of logarithm resistance against reciprocal of temperature yielded larger activation energies for the corrosion process than for ion conduction in the coating, showing that the coating resistance could not be responsible for controlling the corrosion rate. Here further coatings, including a full 3-coat system with sacrificial pigment primer are studied. In this work, the electrochemical activity of the zinc rich primer (coupled to the steel substrate) plus barrier properties of those two topcoats were in concern. Thus, steel with zinc rich primer (ZRP) alone was tested first to identify characteristics of its electrochemical (sacrificial action) behavior.

\section{EXPERIMENTAL PROCEDURE}

A commercial zinc rich primer with $50 \mu \mathrm{m}$ thickness was tested. Coatings were applied to grit-blasted mild steel panels by air spraying. Further tests were conducted on a full 3-coat system consisting of a zinc rich pigmented 2-component epoxy primer $(50 \mu \mathrm{m})$, a low volatile organic content (VOC), high-solid, high-build epoxy barrier coat pigmented with micaceous iron oxide $(180 \mu \mathrm{m})$ and a 2-component acrylic polyurethane topcoat $(60 \mu \mathrm{m})$, prepare and applied similarly. The edges of all panels were protected with a thick highperformance epoxy coating. The electrochemical measurements were made using a three-electrode cell with vertical working electrode of area $40 \mathrm{~cm}^{2}$.The reference electrode was a saturated calomel electrode (SCE) and the counter electrode a platinised titanium electrode of area of 9 $\mathrm{cm}^{2}$. The samples were exposed in hot $3 \% \mathrm{NaCl}$ solution $(800$ $\mathrm{cm}^{3}$ ), made from analytical grade chemicals and deionised water, held at constant temperature in a water bath (or slowly 
cooled in an insulated box). Tests were conducted in duplicate. Impedance measurements were taken at the free corrosion potential using a Gill AC computer-controlled potentiostat with a high-impedance paint buffer (ACM Instruments), using a $20 \mathrm{mV}$ sine amplitude and a frequency range from $0.1 \mathrm{~Hz}$ to $30 \mathrm{kHz}$. Samples were cooled from $50^{\circ} \mathrm{C}$ down to ambient over (typically) $4 \mathrm{~h}$. After the samples had been tested, the cell was replaced in the hot water bath and kept at $50^{\circ} \mathrm{C}$ until the next measurement.

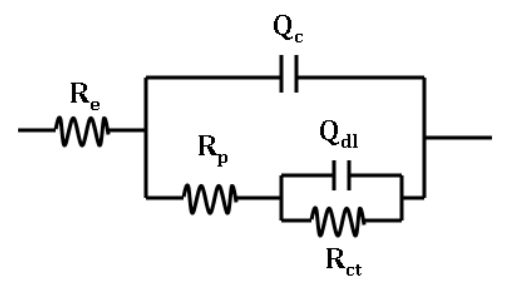

Fig- 1: Model circuit used for fitting

The EIS spectra from zinc rich primer were fitted with circle fit from the ACM software whereas EIS spectra from zinc rich full system coating were fitted to a non-linear least squares fitting software (with ZSimWin) to a R[Q[R[QR]]] model circuit as illustrated in Figure $1[\mathrm{Q}=$ constant phase element $]$.

\section{RESULT AND DISCUSSION}

\subsection{Tests with Zinc Rich Primer (ZRP) Alone}

\subsubsection{Visual Inspection}

Optical micrographs as seen in Figure 2 shows the results of galvanic activity on the surface of the ZRP coating surface before and after exposure up to 22 days in $3 \% \mathrm{NaCl}$ solution. Figure $2 b$ shows the presence of small 'white' particles, probably zinc oxide which starts to build up on the coating surface after 2 days of exposure. By day 19, the presence of blisters was noted and with time, these blisters grow, and unlike normal blisters, they are rough and not smooth (Figure 2c).

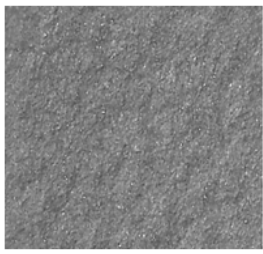

(a)

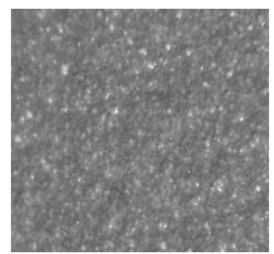

(b)

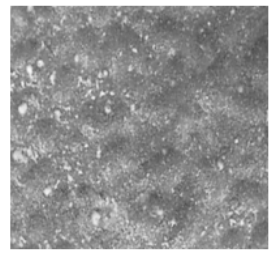

(c)
Fig - 2: Optical image showing the formation of corrosion product on the surface of ZRP coating after (a) 0 day; (b) 2 days; (c) 22 days of exposure

\subsubsection{Preliminary Analysis for Coated Panel Exposed at $21^{\circ} \mathrm{C}$}

After an hour of exposure, Nyquist plot shows two semicircles (Figure 3), similar finding to the study conducted by Meroufel and Touzain [3]. This indicates that the coating is highly porous and permeable. The potentials recorded were -0.96 $\mathrm{V}_{\mathrm{SCE}}$ and $-0.98 \mathrm{~V}_{\mathrm{SCE}}$ which suggests that zinc particles are already starting to corrode.

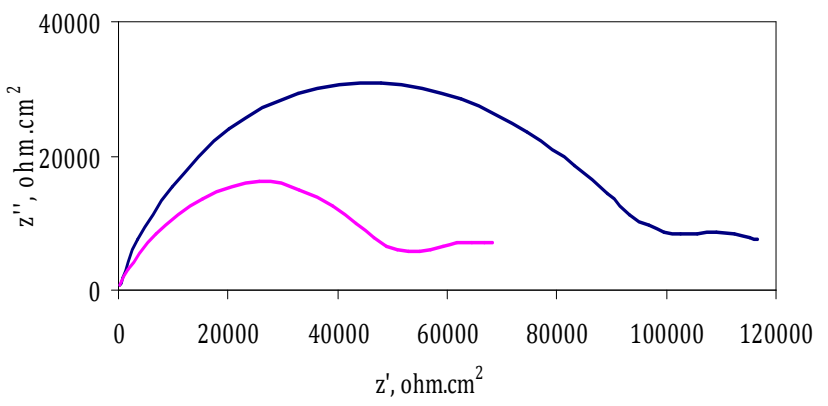

Fig- 3: Nyquist plots obtained for ZRP coating (2 replicates) after 1-hour exposure at ambient temperature

There are disagreements in the literature concerning the analysis and interpretation of the impedance spectra. For some authors, the semicircle at higher frequency corresponds to undefined charge transfer process while the semicircle at low frequency is related to oxygen diffusion $[4,5,6]$. Other studies conclude that semicircle at low frequency is related to the charge transfer for zinc dissolution, whereas the high frequency range shows the dielectric properties of the polymeric matrix [7,8]. An active electrode acts as coating resistance in parallel with double layer capacitance (one semicircle), so for ZRP panels we don't expect two, but sometimes oxide films/passivity generates a more complicated diagram thus the coating does not behave like a simple zinc electrode.

\subsubsection{Open Circuit Potential Measurements}

The duration of protection potential for steel $\left(\mathrm{E}_{\mathrm{pp}}\right)$ is given as being the time during which the potential remains lower than free corrosion potential of steel $\left(-0.65 \mathrm{~V}_{\mathrm{SCE}}\right)$. However it is often taken in practical as being the time which the potential remains lower than $-0.85 \mathrm{~V}_{\mathrm{SCE}}$ [9]. The ZRP coated panel potentials are monitored up to 22 days and presented in Figure 4. 


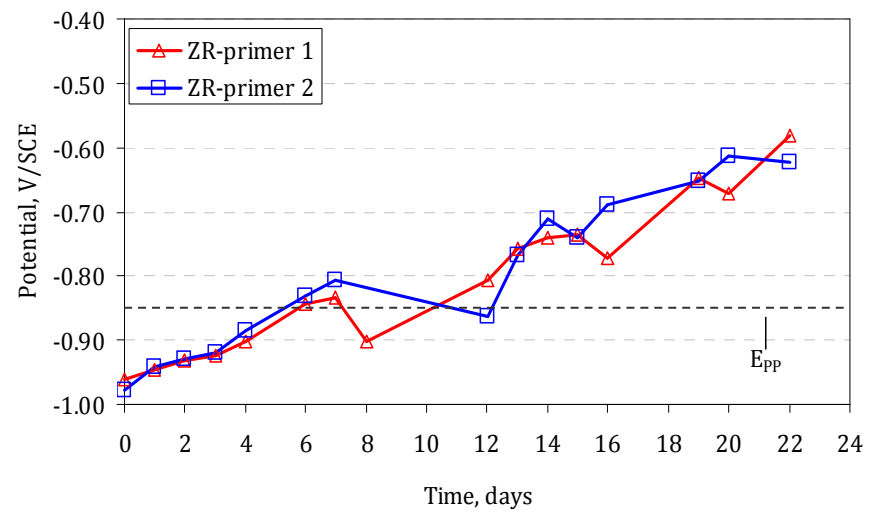

Fig- 4: Evolution of corrosion potential measured at $21^{\circ} \mathrm{C}$ with time for ZRP coating (2 replicates) exposed in $3 \% \mathrm{NaCl}$ solution for 22 days at $50^{\circ} \mathrm{C}$

Due to the high permeability of ZRP coatings, immediately upon immersion in the electrolyte the potential is about $0.98 \mathrm{~V}_{\mathrm{SCE}}$, a value which is close to the potential of zinc in sea water. This indicates clearly that the zinc particles were actively consumed to provide initial galvanic protection to the steel. Notice that the potential passed the $E_{P P}$ value by 6 days. By 22 days, contact between zinc and steel may have been lost as the potential recorded is closed to the corrosion potential of steel.

\subsubsection{Temperature Dependence of Impedance}

The effect of changing temperature on the EIS response of ZRP coating in $3 \% \mathrm{NaCl}$ solution after 7 and 14 days exposure at $50^{\circ} \mathrm{C}$ are shown in Figure 5 and 6 respectively. It seems that the effect of changing temperature is barely noticeable on the impedance spectra after 1 and 4 days of immersion.

However by day 7 the effect of changing temperature becomes much clearer. Even though the effect of temperature on the size of the 'semicircle' is apparent in these figures, Nyquist plots are difficult to fit. Only the first semicircle could be fitted using "circle fit" from the ACM software. Logarithm of coating resistance is then plotted against reciprocal of temperature (Figure 7) and the activation energies measured are in the range of $32-34 \mathrm{kJmol}^{-1}$.

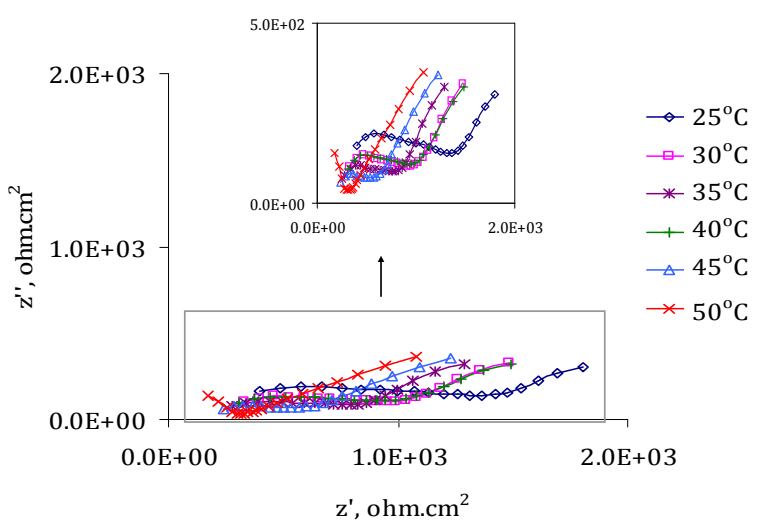

Fig- 5: Nyquist plots for ZRP coating at various temperatures after 7 days of exposure at $50^{\circ} \mathrm{C}$

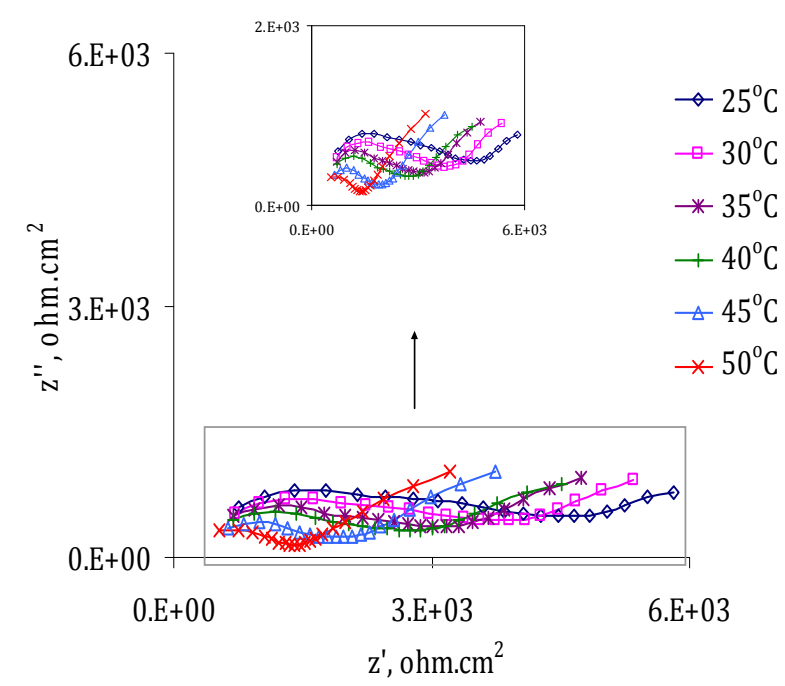

Fig- 6: Nyquist plots for ZRP coating at various temperatures after 14 days of exposure at $50^{\circ} \mathrm{C}$

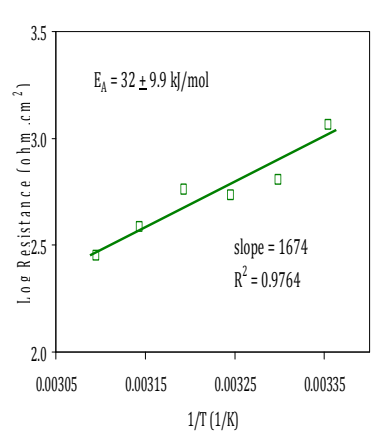

(a)

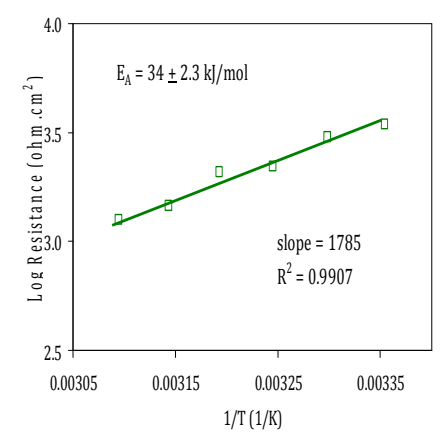

(b)
Fig- 7: Arrhenius plot for ZRP coating after (a) 7 days and (b) 14 days of exposure at $50^{\circ} \mathrm{C}$ 


\subsection{Tests on Full System}

\subsubsection{Open Circuit Potential Measurement}

Figure 8 displays the open circuit potential (OCP) measured over time for coated panels (duplicate sets) exposed at 21 and $50^{\circ} \mathrm{C}$. It is interesting to note that the two panels tested at $21^{\circ} \mathrm{C}$ reacted differently during early immersion.

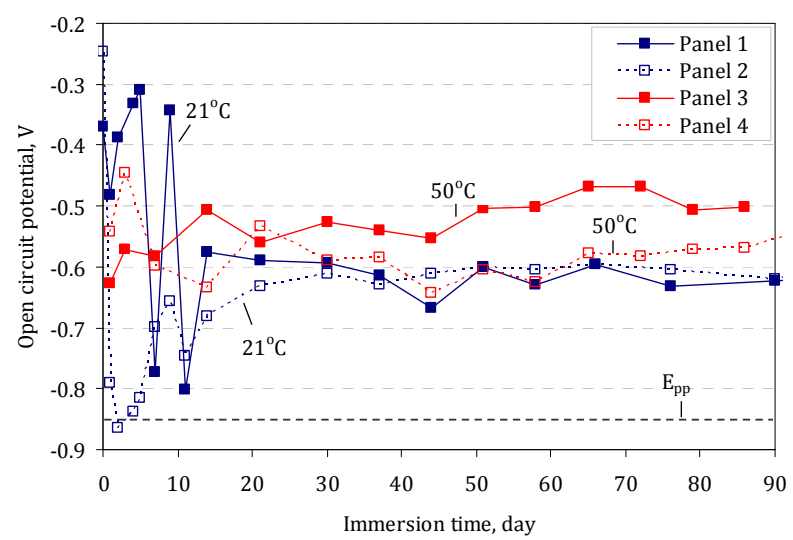

Fig- 8: Evolution of OCP values with immersion time for zinc rich full system coating at $21^{\circ} \mathrm{C}$ and $50^{\circ} \mathrm{C}$

During the first 21 days of immersion Panel 1 had an unstable potential which varied between -0.3 and $-0.8 \mathrm{~V}_{\mathrm{SCE}}$ before reaching a stationary value close to the corrosion potential of steel. In contrast Panel 2 shows potential values close to the zinc potential during early immersion but rapidly increased reaching the corrosion potential of steel by 21 days. Panels 3 and 4 tested at $50^{\circ} \mathrm{C}$ gave comparable potential values. Their values were fluctuating in the range of -0.45 to $-0.65 \mathrm{~V}_{\mathrm{SCE}}$ which is similar to the potential for a coated steel substrate. In this study, the number of days during which the coated panel exhibits more negative potential than $\mathrm{E}_{\mathrm{PP}}\left(-0.85 \mathrm{~V}_{\mathrm{SCE}}\right)$ in $3 \%$ $\mathrm{NaCl}$ solution is used for judgment of how long zinc rich primer coatings provide full cathodic protection to the steel substrate.

According to these results, it is evident that OCP of these panels never lies in cathodic protection region (below $\left.0.85 \mathrm{~V}_{\mathrm{SCE}}\right)$. The potential measured for these coatings will be however a compromise between the zinc-steel galvanic couple (anode and cathode) as suggested by Mayne [10]. Anode potential may give reading at $-0.95 \mathrm{~V}_{\mathrm{SCE}}$ and cathode potential may be a very much higher.

\subsubsection{Effect of Signal Amplitude - A Test of the}

\section{Circuit Model}

ZRP (primer only) gave two semicircles (Figure 3) and here again two semicircles were observed (Figure 9) for the full system. The first semicircle was expected to be the top coating over the zinc rich epoxy primer and the second semicircle corresponded to the electrochemical activity of the zinc rich primer coupled to the steel substrate. To be certain a test is conducted to investigate the effect of changing the signal amplitude on the semicircles as a way of identifying the different features displayed in Nyquist plots. EIS spectra were taken at amplitudes ranging from $20 \mathrm{mV}$ to $120 \mathrm{mV}$. It is clear from Figure 9 that changing the applied potential only changes the shape of the second semicircle.

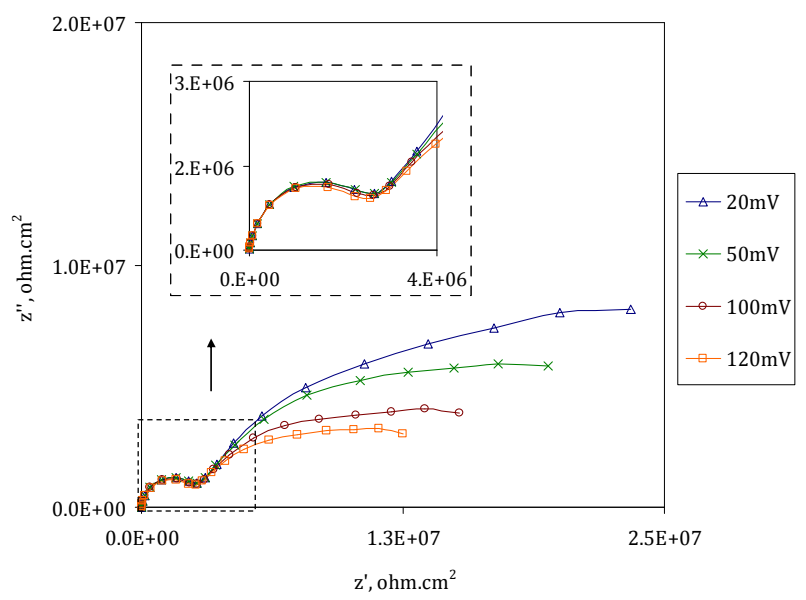

Fig- 9: Nyquist plot at various applied potential for zinc rich full system coating

\subsubsection{Temperature Dependence of Coating}

\section{Parameters}

Figures 10 to 11 show the effect of changing temperature on the EIS response of full system coating after 21 and 35 days exposure at $50^{\circ} \mathrm{C}$. Similar to epoxy-phenolic paint and full system with an inhibitive primer as studied previously $[1,2]$ the size of the semicircles decreases as temperature rises.

These semicircles were fitted to the equivalent circuit in Figure 1 and this procedure give the most accurate determination of coating resistance $\left(R_{p}\right)$ at high frequency and charge transfer resistance $\left(\mathrm{R}_{\mathrm{ct}}\right)$ at low frequency. Then the logarithm of $R_{p}$ and logarithm of $R_{c t}$ were plotted against reciprocal of temperature to determine activation energies for conduction in the coating and the corrosion reaction. A straight line was fitted to the data points in Figure 12 to calculate the activation energies by multiplying the slope with the gas constant, $\mathrm{R}$. 


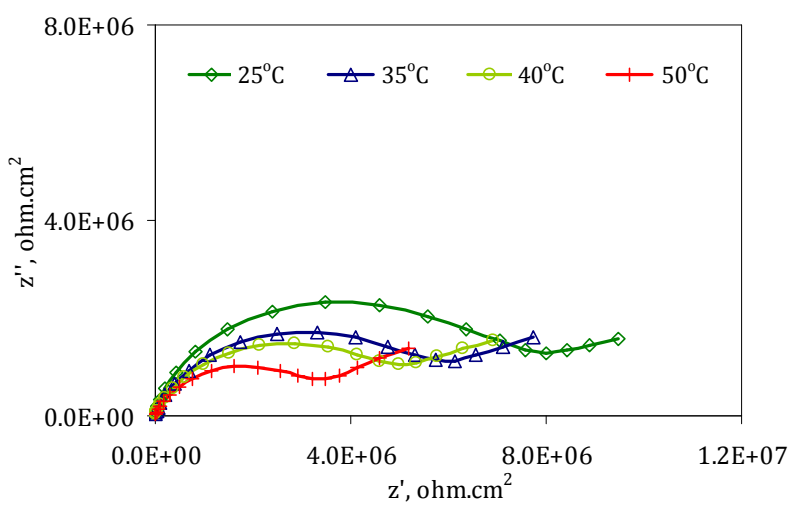

Fig- 10: Nyquist plots at various temperatures for zinc rich full system coating after 21 days of exposure at $50^{\circ} \mathrm{C}$

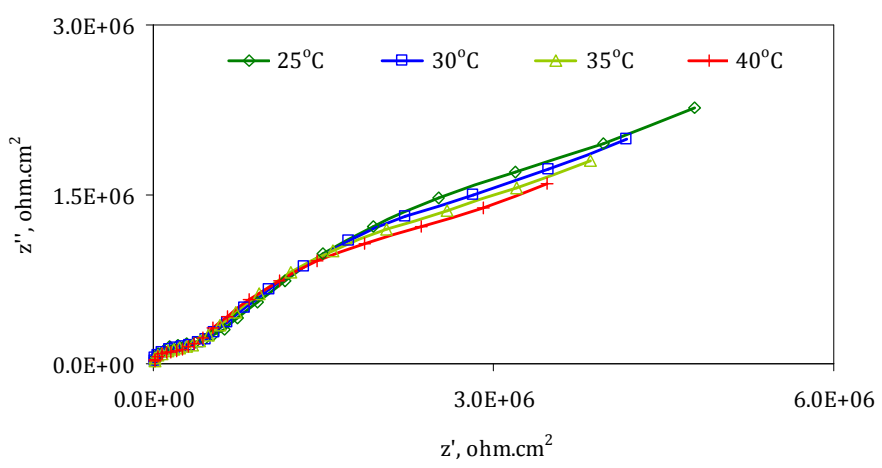

Fig- 11: Nyquist plots at various temperatures for zinc rich full system coating after 35 days of exposure at $50^{\circ} \mathrm{C}$

The effect of temperature on ion transport through the film and the corrosion process are summarized in Figure 12 which shows the trend of activation energy determined for ion conduction in the film $\left(R_{p}\right)$ and corrosion process (from $R_{c t}$ ). The activation energy for the corrosion process is very much higher than ion conduction during earlier immersion.

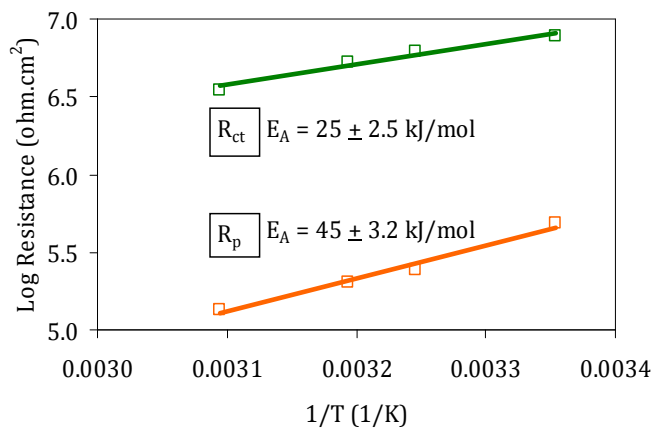

(a)

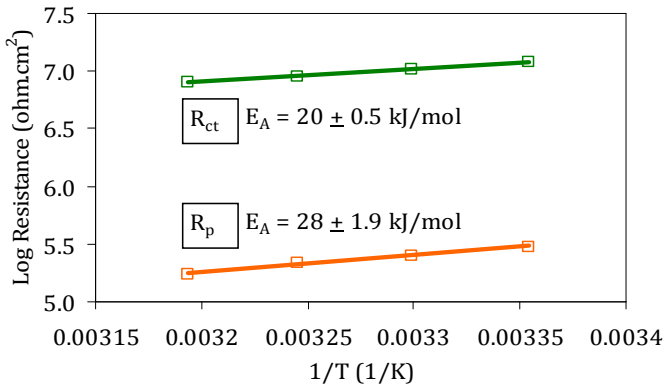

(b)

Fig- 12: Arrhenius plots of $R_{p}$ and $R_{c t}$ for zinc rich full system coating after (a) 21 days and (b) 35 days of exposure at $50^{\circ} \mathrm{C}$

It is evident from Figure 13 that the activation energy for the corrosion process decreasing overtime, and at the end of the exposure, they are quite similar. It is suggested that at this stage ion transport in the coating might be controlling the corrosion process. In the beginning the activation energy values get smaller over time because of coating degradation.

If we compare these result with zinc phosphate full system coating as reported previously $[1,2,11]$ we see that $R_{p}$ values for 2 coats of zinc rich full system are similar to those for the first semi-circles on the thicker zinc phosphate full system coating; suggesting that the interpretation offered here is correct. However the high activation energy values seen in the zinc phosphate full system coating were not seen here.
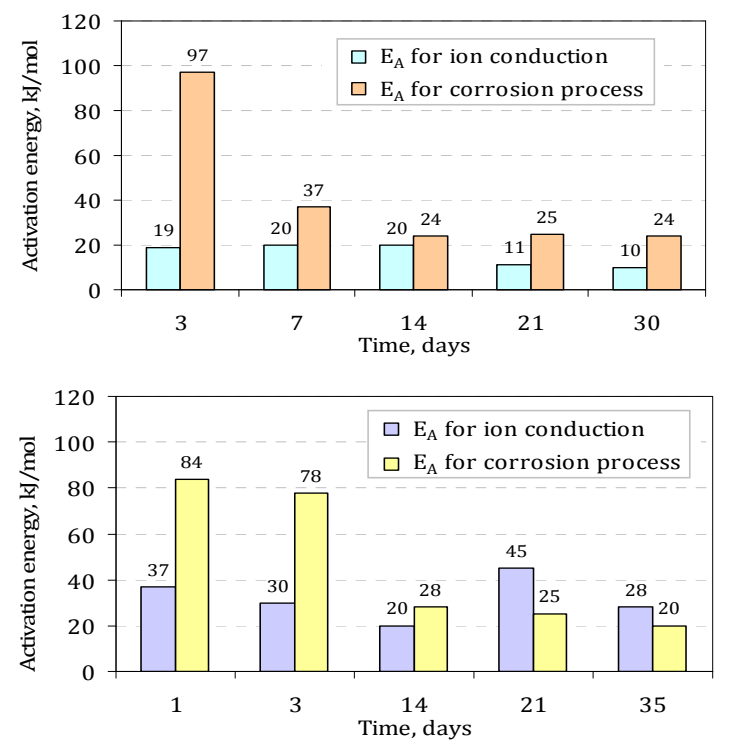

Fig- 13: Evolution with time of activation energy for ion conduction and corrosion process for zinc rich full system coating (duplicate sets) 


\section{CONCLUSIONS}

In contrast with the zinc phosphate full system coating [1], zinc rich full system gave the same conclusion as the thinner coatings [2] where the coating resistance from EIS cannot be controlling the corrosion reaction. The activation energies generated for the corrosion process here (78-97 kJmol-1) are very much higher than those of ion transport through the coating (19-37 kJmol-1) during early immersion (Figure 13).

Further interesting findings come from the activation energy trends over time particularly for the corrosion process where the value is decreasing, so that at the end of exposure the values for Rp and Rct become quite similar. It is suggested that at this stage ion transport in the coating might be controlling the corrosion process unlike at the beginning; the activation energy is getting smaller due to coating degradation.

\section{ACKNOWLEDGEMENTS}

We are grateful to Ministry of Higher Education of Malaysia for awarding the scholarship that supported this research and Shell Global Solution International BV for providing samples and financial support.

\section{REFERENCES}

[1] Z. Sharer Sahir, J.M. Sykes, "Effect of Temperature on the Impedance Response of Coated Metals, Application of Electrochemical to Organic Coating, Conference Paper, Switzerland (2013)

[2] Z. Sharer, J. Sykes, "Insights into Protection Mechanisms of Organic Coatings from Thermal Testing with EIS", Progress in Organic Coatings, 74, 2 (2012), p. 405-409

[3] A. Meroufel, S.Touzain, "EIS Characterization of New Zinc-Rich Powder Coatings" Journal of Progress in Organic Coatings, 59, 3 (2007), p. 197-205

[4] S. Feliu, Jr., R. Barajas, J. M. Bastidas, M. Morcillo, S. Felliu, "Study of Protection Mechanism of Zinc Rich Paints by Electrochemical Impedance Spectroscopy", Electrochemical Impedance: Analysis and Interpretation, ASTM STP 1188, J.R. Scully, D.C. Silverman and M.W. Kending (eds) American Society for Testing and Materials, Philadelphia (1993) p. 438-449

[5] D. Pereira, J. D. Scantlebury, M. G. S. Ferreira, M. E. Almeida, "The Application of Electrochemical Measurements to the Study and Behaviour of Zinc-Rich Coatings" Journal of Corrosion Science, 30, 11 (1990), p. 1135-1147

[6] S. E. Faidi, J. D. Scantlebury, P. Bullivant, N. T. Whittle, R. Savin, "An Electrochemical Study of Zinc-Containing Epoxy Coatings on Mild Steel", Journal of Corrosion Science, 35,5-8 (1993), p. 1319-1328

[7] X.R. Novoa, M. IZquierdo, P. Merino, L. Espada, "Electrical Impedance Spectroscopy and Zero Resistance Ammeter (ZRA) as Tools for Studying the Behaviour of ZincRich Inorganic Coatings", Materials Science Forum, 44\&45 (1989) p. $223-234$
[8] C.A. Gervasi, A. R. Di Sarli, E. Cavalcanti, O. Ferraz, E.C. Bucharsky, S. G. Real, J. R. Vilche, "The Corrosion Protection of Steel in Sea Water using Zinc-Rich Alkyd Paints. An Assessment of the Pigment-Content Effect by EIS", Journal of Corrosion Science, 36, 12 (1994), p. 1963-1972

[9] C. M. Abreu, M. Izquierdo, M. Keddam, X. R. Nóvoa, H. Takenouti, H. "Electrochemical Behaviour of Zinc-Rich Epoxy Paints in 3\% NaCl Solution", Electrochimica Acta, 41, 15 (1996) p. 2405-2415

[10] J.E.O. Mayne, "Electrochemical Behaviour of Paint Films in Sea-Water, Chemistry and Industry (1951)

[11] Z. Sharer Sahir, "Investigation of Protective of Organic Coatings by Thermal Testing and Electrochemical Techniques”, DPhil Thesis, University of Oxford, 2011 